\title{
A STUDY OF THE COUPLED FIXED POINT PROBLEM FOR OPERATORS SATISFYING A MAX-SYMMETRIC CONDITION IN $b$-METRIC SPACES WITH APPLICATIONS TO A BOUNDARY VALUE PROBLEM
}

\author{
ADRIAN PETRUŞEL, GABRIELA PETRUŞEL, AND BESSEM SAMET \\ Received 12 November, 2015
}

\begin{abstract}
In this paper, we will consider the coupled fixed point problem for single-valued operators satisfying a symmetric contraction condition with respect to maximum. An application to a periodic boundary value problem illustrates the results. The study of a coupled coincidence problem is also suggested.
\end{abstract}

2010 Mathematics Subject Classification: 47H10; 54H25; 34B15

Keywords: single-valued operator, $b$-metric space, fixed point, ordered metric space, coupled fixed point, data dependence, well-posedness, Ulam-Hyers stability, limit shadowing, periodic boundary value problem

\section{INTRODUCTION}

An interesting extension of the Banach's contraction principle was given, in the framework of so-called $b$-metric spaces (or quasimetric spaces), by S. Czerwik, see [5]. For some previous results concerning the topology of $b$-metric spaces see also I.A. Bakhtin [1], L.M. Blumenthal [4], J. Heinonen [8] and the references therein.

The concept of coupled fixed point and the study of coupled fixed point problems appeared, for the first time, in some papers of Opoitsev (see [11]), and then in the paper-source of D. Guo and V. Lakshmikantham [7], where the monotone iteration technique is exploited. Then, T. Gnana Bhaskar and V. Lakshmikantham in [6] considered the contraction type method and gave important abstract results and nice applications to periodic boundary value problems. For other contributions, see [10], [15], [16] and the references therein.

The first author thanks the Visiting Professor Programming at King Saud University for funding this work. The third author extends his sincere appreciation to the Deanship of Scientific Research at King Saud University for its funding this Prolific Research Group (PRG-1436-10).

(c) 2016 Miskolc University Press 
If $(X, d)$ is a metric space and $T: X \times X \rightarrow X$ is an operator, then, by definition, a coupled fixed point for $T$ is a pair $\left(x^{*}, y^{*}\right) \in X \times X$ satisfying

$$
\left\{\begin{array}{c}
x^{*}=T\left(x^{*}, y^{*}\right) \\
y^{*}=T\left(y^{*}, x^{*}\right)
\end{array}\right.
$$

We will denote by $C F i x(T)$ the coupled fixed point set for $T$.

Some nice generalizations of the results given in [6] were presented by V. Berinde in [3], where a symmetric contraction type condition on the operator $T$ is assumed. See also [12] for a similar approach, but using a different metric.

The aim of this paper is to present, in the context of $b$-metric spaces, some coupled fixed point theorems for another type of symmetric contractions with respect to the maximum. An application to a boundary value problem, via a system of integral equations will illustrate the theory.

\section{PRELIMINARIES}

Throughout this paper $\mathbb{N}$ stands for the set of natural numbers, while $\mathbb{N}^{*}$ for the set of natural numbers except 0 .

We will recall now the definition of a $b$-metric space.

Definition 1 (Bakhtin [1], Czerwik [5]). Let $X$ be a nonempty set and let $s \geq 1$ be a given real number. A functional $d: X \times X \rightarrow \mathbb{R}_{+}$is said to be a $b$-metric if all the axioms of the metric are satisfied, with the following exception:

$$
d(x, z) \leq s[d(x, y)+d(y, z)], \text { for all } x, y, z \in X .
$$

A pair $(X, d)$ with the above properties is called a $b$-metric space.

Some examples of $b$-metric spaces are given in [2], [5], ...

It is worth to mention that the $b$-metric on a nonempty set $X$ need not be continuous. Moreover, open balls in such spaces need not be open sets in the topology induced by $d$. The convergence of sequences is defined in the classical way, i.e., $\left(x_{n}\right)_{n \in \mathbb{N}} \subset X$ converges to $x \in X$ if $\left(d\left(x_{n}, x\right)\right)_{n \in \mathbb{N}} \rightarrow 0$ as $n \rightarrow \infty$. In this context, a set $Y \subset X$ is said to be closed if for any sequence $\left(x_{n}\right)_{n \in \mathbb{N}}$ in $Y$ which is convergent to some $x$, we have that $x \in Y$.

Lemma 1. Let $(X, d)$ be a $b$-metric space with constant $s \geq 1$. Then, the functional $\tilde{d}:(X \times X) \times(X \times X) \rightarrow \mathbb{R}_{+}$defined by

$$
\tilde{d}((x, y),(u, v)):=\max \{d(x, u), d(y, v)\}
$$

is a b-metric on $X \times X$ with the same constant $s \geq 1$.

Proof. We will only establish the third axiom of the $b$-metric. We have to prove that, for every $(x, y),(u, v),(a, b)) \in X \times X$, we have:

$$
\tilde{d}((x, y),(u, v)) \leq s(\tilde{d}((x, y),(a, b))+\tilde{d}((a, b),(u, v))),
$$


which means that

$$
\max \{d(x, u), d(y, v)\} \leq s(\max \{d(x, a), d(y, b)\}+\max \{d(a, u), d(b, v)\}) .
$$

Since $d$ is a $b$-metric on $X$, we know that

$$
d(x, u) \leq s(d(x, a)+d(a, u)) \text { and } d(y, v) \leq s(d(y, b)+d(b, v)) .
$$

Thus, using the inequality between positive real numbers

$$
\max \{\alpha+\beta, \gamma+\delta\} \leq \max \{\alpha, \gamma\}+\max \{\beta, \delta\},
$$

we immediately get the conclusion.

We also mention two continuity concepts. Let $(X, d)$ be a $b$-metric space. Then $f$ is called:

a) continuous on $X$ if for every $x \in X$ and any sequence $\left(x_{n}\right)_{n \in \mathbb{N}}$ in $X$ which converges to $x$ in $(X, d)$, it follows that the sequence $\left(f\left(x_{n}\right)\right)_{n \in \mathbb{N}}$ converges to $f(x)$ in $(X, d)$;

b) with closed graph if for every sequence $\left(x_{n}\right)_{n \in \mathbb{N}}$ in $X$ which converges to $x$ in $(X, d)$ and the sequence $\left(f\left(x_{n}\right)\right)_{n \in \mathbb{N}}$ converges to $y$ in $(X, d)$ as $n \rightarrow \infty$, we have that $y=f(x)$.

If $X$ is a nonempty set and $f: X \rightarrow X$, then we denote Fix $(f):=\{x \in X: x=$ $f(x)\}$, the fixed point set for $f$ and by $\operatorname{Graph}(f):=\{(x, f(x)) \mid x \in X\}$, the graph of $f$.

\section{EXISTENCE AND UNIQUENESS RESULTS FOR THE COUPLED FIXED POINT PROBLEM WITH MAX-SYMMETRIC CONTRACTION TYPE OPERATORS}

We will present first a coincidence point result which is an extension to the case of $b$-metric spaces and to a coincidence point problem of a well known fixed point theorem given by Ran and Reurings [14].

Theorem 1. Let $(X, d)$ be a b-metric space with constant $\lambda \geq 1, Y$ be a nonempty set and " $\leq$ " be a partial order relation on $Y$. Let $\rho$ be a b-metric on $Y$ with constant $s \geq 1$ and $g, t: X \rightarrow Y$ be two operators which have closed graph. Suppose that:

(i) $t(X) \subset g(X)$;

(ii) $(t(X), \rho)$ is a complete subset of $Y$;

(i) there exists $k \in\left(0, \frac{1}{s}\right)$ such that $d(t(x), t(y)) \leq k d(g(x), g(y)), \forall x, y \in$ $X$ with $g(x) \leq g(y)$;

(ii) there is $x_{0} \in X$ such that $g\left(x_{0}\right) \in t(X)$ and $g\left(x_{0}\right) \leq t\left(x_{0}\right)$;

(iii) $t$ is increasing wit respect to $g$, i.e.,

$$
x_{1}, x_{2} \in X \text { and } g\left(x_{1}\right) \leq g\left(x_{2}\right) \Rightarrow t\left(x_{1}\right) \leq t\left(x_{2}\right) .
$$

Then, there exists $x^{*} \in X$ and $y^{*} \in t(X)$ such that $g\left(x^{*}\right)=t\left(x^{*}\right)=y^{*}$ and, moreover, the sequence $\left(z_{n}\right)_{n \in \mathbb{N}}$ defined by $g\left(z_{n+1}\right)=t\left(z_{n}\right)$, starting from any point $z_{0} \in X$ which is comparable to $x_{0}$, converges to a coincidence point of $t$ and $g$. 
Proof. Let us define $f:=t \circ g^{-1}$. Then, we have for $f$ the following properties:

1) $f$ is a single-valued operator on $t(X)$;

2) $f: t(X) \rightarrow t(X)$;

3) $f$ has closed graph;

3) $\rho\left(f\left(y_{1}\right), f\left(y_{2}\right)\right) \leq k \rho\left(y_{1}, y_{2}\right)$, for all $y_{1}, y_{2} \in t(X)$ with $y_{1} \leq y_{2}$;

4) $f$ is increasing on $t(X)$;

5) if $y_{0}:=g\left(x_{0}\right)$, then $y_{0} \leq\left(t \circ g^{-1}\right)\left(y_{0}\right)=f\left(y_{0}\right)$.

By Theorem 3.1 in [12], we obtain that $\operatorname{Fix}(f) \neq \varnothing$. Let $y^{*} \in$ Fix $(f)$. Then $\left(t \circ g^{-1}\right)\left(y^{*}\right)=y^{*}$. Thus, if we denote $x^{*}:=g^{-1}\left(y^{*}\right)$, then we have $t\left(x^{*}\right)=$ $g\left(x^{*}\right)=y^{*}$, showing that $x^{*}$ is a coincidence point for $t$ and $g$. Moreover, the sequence $y_{n+1}=f\left(y_{n}\right)$ (where $n \in \mathbb{N}$ ), starting from $y_{0}:=t\left(x_{0}\right)$ converges to $y^{*}$ as $n \rightarrow \infty$, while the sequence $x_{n}$ defined by $g\left(x_{n+1}\right)=t\left(x_{n}\right)$ (where $n \in \mathbb{N}$ ) converges to $x^{*}$ as $n \rightarrow \infty$. The final part of the conclusion follows from Theorem 3.1 in [12].

Remark 1. Notice that, as in the fixed point equation case, the uniqueness of the fixed point of $f$ and global convergence of the successive approximations sequence of $f$ (and, as a consequence, that of the coincidence point) can be obtained adding the hypotheses that $g$ is an injection and every pair of elements of $X$ has a lower bound or an upper bound (or an equivalent assumption that for every $x, y \in X$ there exists $z \in X$ which is comparable to $x$ and $y$ ).

Remark 2. The particular case $X=Y$ and $g(x)=x$, leads to Theorem 3.1 in [12].

We recall now the concept of mixed monotone operator.

Definition 2. Let $(X, \leq)$ a partially ordered set and $T: X \times X \rightarrow X$. We say that $T$ has the mixed monotone property if $T(\cdot, y)$ is monotone increasing for any $y \in X$ and $T(x, \cdot)$ is monotone decreasing for any $x \in X$.

Let $(X, \preceq)$ be a partially ordered set and $d$ be a $b$-metric on $X$. Notice that we can endow the product space $X \times X$ with the following partial order:

$$
\text { for }(x, y),(u, v) \in X \times X \text {, we write }(x, y) \leq_{P}(u, v) \Leftrightarrow x \preceq u, y \succeq v \text {. }
$$

Our first existence result for the coupled fixed point problem (1.1) is the following.

Theorem 2. Let $(X, \preceq)$ be a partially ordered set and let $d: X \times X \rightarrow \mathbb{R}_{+}$be a complete b-metric on $X$ with constant $s \geq 1$. Let $T: X \times X \rightarrow X$ be an operator with closed graph which has the mixed monotone property on $X \times X$. Assume that the following conditions are satisfied:

(i) there exists $k \in\left(0, \frac{1}{s}\right)$ such that $\max \{d(T(x, y), T(u, v)), d(T(y, x), T(v, u))\} \leq k \max \{d(x, u), d(y, v)\}, \forall x \preceq u, y \succeq v$;

(ii) there exist $x_{0}, y_{0} \in X$ such that $x_{0} \preceq T\left(x_{0}, y_{0}\right)$ and $y_{0} \succeq T\left(y_{0}, x_{0}\right)$; 
Then, the following conclusions hold:

(a) there exists $\left(x^{*}, y^{*}\right) \in X \times X$ a solution of the coupled fixed point problem (1.1), such that the sequences $\left(x_{n}\right)_{n \in \mathbb{N}},\left(y_{n}\right)_{n \in \mathbb{N}}$ in $X$ defined, for $n \in \mathbb{N}$, by

$$
\left\{\begin{array}{l}
x_{n+1}=T\left(x_{n}, y_{n}\right) \\
y_{n+1}=T\left(y_{n}, x_{n}\right)
\end{array}\right.
$$

have the property that $\left(x_{n}\right)_{n \in \mathbb{N}} \rightarrow x^{*},\left(y_{n}\right)_{n \in \mathbb{N}} \rightarrow y^{*}$ as $n \rightarrow \infty$. Moreover, for every pair $(x, y) \in X \times X$ with $x \preceq x_{0}$ and $y \succeq y_{0}$ (or reversely), we have that $\left(T^{n}(x, y)\right)_{n \in \mathbb{N}}$ converges to $x^{*}$ and $\left(T^{n}(y, x)\right)_{n \in \mathbb{N}}$ converges to $y^{*}$. holds

(b) In particular, if the b-metric $d$ is continuous, then the following estimation

$$
\max \left\{d\left(T^{n}\left(x_{0}, y_{0}\right), x^{*}\right), d\left(T^{n}\left(y_{0}, x_{0}\right), y^{*}\right)\right\} \leq \frac{s k^{n}}{1-s k} \cdot \max \left\{d\left(x_{0}, T\left(x_{0}, y_{0}\right)\right), d\left(y_{0}, T\left(y_{0}, x_{0}\right)\right)\right\},
$$

for all $n \in \mathbb{N}^{*}$.

Proof. We denote $Z:=X \times X$. By (ii), we have that $z_{0}:=\left(x_{0}, y_{0}\right) \leq_{P}\left(x_{1}, y_{1}\right):=$ $z_{1}$. If we define $x_{2}:=T\left(x_{1}, y_{1}\right)$ and $y_{2}:=T\left(y_{1}, x_{1}\right)$, then we get

$$
x_{2}:=T\left(x_{1}, y_{1}\right)=T^{2}\left(x_{0}, y_{0}\right) \text { and } y_{2}:=T\left(y_{1}, x_{1}\right)=T^{2}\left(y_{0}, x_{0}\right) .
$$

With these notations, due to the mixed monotone property of $T$, we have that

$$
x_{2}=T\left(x_{1}, y_{1}\right) \succeq T\left(x_{0}, y_{0}\right)=x_{1} \text { and } y_{2}=T\left(y_{1}, x_{1}\right) \preceq T\left(y_{0}, x_{0}\right)=y_{1},
$$

which means that $z_{1}=\left(x_{1}, y_{1}\right) \leq_{P}\left(x_{2}, y_{2}\right):=z_{2}$.

By this approach, we obtain the sequences $\left(x_{n}\right)_{n \in \mathbb{N}},\left(y_{n}\right)_{n \in \mathbb{N}}$ in $X$ with

$$
\left\{\begin{array}{l}
x_{n+1}=T\left(x_{n}, y_{n}\right) \\
y_{n+1}=T\left(y_{n}, x_{n}\right),
\end{array}\right.
$$

By induction, we can easily verify that

$$
z_{n}:=\left(x_{n}, y_{n}\right) \leq_{P}\left(x_{n+1}, y_{n+1}\right):=z_{n+1}, \forall n \in \mathbb{N} .
$$

Hence, $\left(z_{n}\right)_{n \in \mathbb{N}}$ is a monotone increasing sequence in $\left(Z, \leq_{P}\right)$.

We introduce now the functional $\tilde{d}: Z \times Z \rightarrow \mathbb{R}_{+}$defined by

$$
\tilde{d}((x, y),(u, v)):=\max \{d(x, u), d(y, v)\} .
$$

By Lemma 1 , the functional $\tilde{d}$ defines a $b$-metric on $Z$ with the same constant $s \geq 1$ and, moreover, if the space $(X, d)$ is complete, then $(Z, \tilde{d})$ is complete too.

We define on $Z$ the operator $F: Z \rightarrow Z$ given by

$$
F(x, y):=(T(x, y), T(y, x)) .
$$

Notice first that $z_{n+1}=F\left(z_{n}\right)$, for $n \in \mathbb{N}$, where $z_{0}:=\left(x_{0}, y_{0}\right)$. Secondly, let us observe that, by the mixed monotone property of $T$, we have that $F$ is monotone increasing with respect to $\leq_{P}$, i.e.,

$$
(x, y),(u, v) \in Z \text {, with }(x, y) \leq_{P}(u, v) \Rightarrow F(x, y) \leq_{P} F(u, v) .
$$

Notice also that, since $T$ has closed graph, then $F$ has closed graph too. 
We prove now that $F$ is a contraction in $(Z, \tilde{d})$ on all comparable (with respect to $\left.\leq_{P}\right)$ elements of $Z$. Indeed, for all $z, w \in Z$ with $z:=(x, y) \leq_{P}(u, v):=w$, we have

$\tilde{d}(F(z), F(w))=\tilde{d}(F(x, y), F(u, v))=\tilde{d}((T(x, y), T(y, x)),(T(u, v), T(v, u)))=$ $\max \{d(T(x, y), T(u, v)), d(T(y, x), T(v, u))\} \leq k \max \{d(x, u), d(y, v)\}=k \tilde{d}(z, w)$.

Moreover, since

$$
\tilde{d}\left(F\left(z_{n}\right), F\left(z_{n+1}\right)\right) \leq k^{n} \tilde{d}\left(z_{0}, z_{1}\right),
$$

we have that

$$
\tilde{d}\left(z_{n}, z_{n+p}\right) \leq s k^{n}\left(1+s k+\cdots+(s k)^{p-1}\right) \tilde{d}\left(z_{0}, z_{1}\right)=s k^{n} \frac{1-(s k)^{p}}{1-s k} \tilde{d}\left(z_{0}, z_{1}\right) .
$$

Thus, by our hypotheses and the definition of $F$, we get that $F$ satisfies all the assumptions in Theorem 1 (for the particular case presented in Remark 2). Hence we get that $F$ has at least one fixed point $z^{*} \in Z$ and, for any $z \in Z$ which is comparable with $z_{0}$, the sequence of successive approximations for $F$ starting from $z$ converges to a fixed point of $F$. In particular, the sequence $z_{n}=\left(x_{n}, y_{n}\right)$ constructed below converges in $(Z, \tilde{d})$ to $z^{*}:=\left(x^{*}, y^{*}\right)$ as $n \rightarrow \infty$ and, if additionally, the $b$-metric $d$ is continuous, we have the following estimation of the error:

$$
\tilde{d}\left(z_{n}, z^{*}\right) \leq \frac{s k^{n}}{1-s k} \cdot \tilde{d}\left(z_{0}, z_{1}\right), \text { for all } n \in \mathbb{N}^{*},
$$

which means that

$$
\max \left\{d\left(x_{n}, x^{*}\right), d\left(y_{n}, y^{*}\right)\right\} \leq \frac{s k^{n}}{1-s k} \cdot \max \left\{d\left(x_{0}, x_{1}\right), d\left(y_{0}, y_{1}\right)\right\} \text {, for all } n \in \mathbb{N}^{*} \text {. }
$$

As a consequence, we also have that $\left(x_{n}\right)_{n \in \mathbb{N}} \rightarrow x^{*},\left(y_{n}\right)_{n \in \mathbb{N}} \rightarrow y^{*}$ in $(X, d)$ as $n \rightarrow$ $\infty$. Moreover, by the particular case of Theorem 1 noticed in Remark 2, for every pair $(x, y) \in X \times X$ with $x \leq x_{0}$ and $y \geq y_{0}$ (or reversely), we have that $\left(T^{n}(x, y)\right)_{n \in \mathbb{N}}$ converges to $x^{*}$ and $\left(T^{n}(y, x)\right)_{n \in \mathbb{N}}$ converges to $y^{*}$. Notice also, that by (3.3), letting $p \rightarrow \infty$, we get the final estimation of the conclusion. This completes the proof.

Concerning the uniqueness of the coupled fixed point, by Remark 1, we get the following result.

Theorem 3. If, in addition to the hypotheses of Theorem 2, we suppose either that for every $(x, y),(u, v) \in X \times X$ there exists $(z, w) \in X \times X$ such that

$$
\left\{\begin{array}{l}
(x \preceq z, y \succeq w) \text { or }(z \preceq x, w \succeq y) \\
(u \preceq z, v \succeq w) \text { or }(z \preceq u, w \succeq v),
\end{array}\right.
$$

or that

$$
\text { every pair of elements of } X \text { has a lower bound or an upper bound, }
$$


then the coupled fixed point in Theorem 2 is unique and for every pair $(x, y) \in X \times X$ we have that $\left(T^{n}(x, y)\right)_{n \in \mathbb{N}}$ converges to $x^{*}$ and $\left(T^{n}(y, x)\right)_{n \in \mathbb{N}}$ converges to $y^{*}$ as $n \rightarrow \infty$.

Concerning the existence of a fixed point for $T$ (in the sense that $T(x, x)=x$ ), we can prove the following result.

Theorem 4. If we assume that all the hypotheses of Theorem 3 take place, then for the unique coupled fixed point $\left(x^{*}, y^{*}\right)$ of $T$ we have that $x^{*}=y^{*}$, i.e., $x^{*}$ is a fixed point for $T$.

Proof. We will work again on $Z$ with the metric $\tilde{d}: Z \times Z \rightarrow \mathbb{R}_{+}$defined by

$$
\tilde{d}((x, y),(u, v)):=\max \{d(x, u), d(y, v)\} .
$$

From Theorem 3, the coupled fixed point problem for $T$ has a unique solution $\left(x^{*}, y^{*}\right)$. We will consider two cases:

Case 1. If $x^{*}$ and $y^{*}$ are comparable, then, from the contraction condition on $T$, written for $x=v:=x^{*}, y=u=y^{*}$, we obtain

$$
\max \left\{d\left(T\left(x^{*}, y^{*}\right), T\left(y^{*}, x^{*}\right)\right), d\left(T\left(y^{*}, x^{*}\right), T\left(x^{*}, y^{*}\right)\right)\right\} \leq k \max \left\{d\left(x^{*}, y^{*}\right), d\left(y^{*}, x^{*}\right)\right\},
$$

which yields that $d\left(x^{*}, y^{*}\right) \leq k d\left(x^{*}, y^{*}\right)$. Since $k<1$, we get that $d\left(x^{*}, y^{*}\right)=0$. Thus $x^{*}=y^{*}$.

Case 2. If $x^{*}$ and $y^{*}$ are not comparable, then there exists $z \in X$ which is comparable to $x^{*}$ and $y^{*}$. Suppose, for example, that $x^{*} \preceq z$ and $y^{*} \preceq z$. In view of the definition of the partially order relation $\leq_{P}$ on $X \times X$, we obtain that $\left(x^{*}, y^{*}\right)$, $\left(x^{*}, z\right),\left(z, x^{*}\right)$ and $\left(y^{*}, x^{*}\right)$ are comparable with respect to $\leq_{P}$. From the proof of Theorem 2, we know that $F: X \times X \rightarrow X \times X$, given by $F(x, y)=(T(x, y), T(y, x))$ is a $k$-contraction on all comparable (with respect to $\leq_{P}$ ) elements of $Z$. Moreover

$$
\tilde{d}\left(F^{n}(u), F^{n}(v)\right) \leq k^{n} \tilde{d}(u, v), \forall u, v \in X \times X \text {, with } u \leq_{P} v .
$$

Then, for $u:=\left(x^{*}, z\right)$ and $v:=\left(x^{*}, y^{*}\right)$, we get

$$
\tilde{d}\left(F^{n}\left(x^{*}, z\right), F^{n}\left(x^{*}, y^{*}\right)\right) \leq k^{n} \tilde{d}\left(\left(x^{*}, z\right),\left(x^{*}, y^{*}\right)\right)=k^{n} d\left(z, y^{*}\right) .
$$

Similarly, for $u:=\left(x^{*}, z\right)$ and $v:=\left(z, x^{*}\right)$, we obtain

$$
\tilde{d}\left(F^{n}\left(x^{*}, z\right), F^{n}\left(z, x^{*}\right)\right) \leq k^{n} \tilde{d}\left(\left(x^{*}, z\right),\left(z, x^{*}\right)\right)=k^{n} d\left(x^{*}, z\right),
$$

while for $u:=\left(y^{*}, x^{*}\right)$ and $v:=\left(z, x^{*}\right)$, we can write that

$$
\tilde{d}\left(F^{n}\left(y^{*}, x^{*}\right), F^{n}\left(z, x^{*}\right)\right) \leq k^{n} \tilde{d}\left(\left(y^{*}, x^{*}\right),\left(z, x^{*}\right)\right)=k^{n} d\left(y^{*}, z\right) .
$$

As a consequence of the above three relations, we have

$$
\begin{gathered}
d\left(x^{*}, y^{*}\right)=\tilde{d}\left(\left(x^{*}, y^{*}\right),\left(y^{*}, x^{*}\right)\right)=\tilde{d}\left(F^{n}\left(x^{*}, y^{*}\right), F^{n}\left(y^{*}, x^{*}\right)\right) \leq \\
s\left(\tilde{d}\left(F^{n}\left(x^{*}, y^{*}\right), F^{n}\left(x^{*}, z\right)\right)+\tilde{d}\left(F^{n}\left(x^{*}, z\right), F^{n}\left(y^{*}, x^{*}\right)\right)\right) \leq \\
s \tilde{d}\left(F^{n}\left(x^{*}, y^{*}\right), F^{n}\left(x^{*}, z\right)\right)+s^{2}\left(\tilde{d}\left(F^{n}\left(x^{*}, z\right), F^{n}\left(z, x^{*}\right)\right)+\tilde{d}\left(F^{n}\left(z, x^{*}\right), F^{n}\left(y^{*}, x^{*}\right)\right)\right) \leq
\end{gathered}
$$


$s k^{n} d\left(z, y^{*}\right)+s^{2} k^{n}\left(d\left(x^{*}, z\right)+d\left(y^{*}, z\right)\right)=s k^{n}\left[(1+s) d\left(y^{*}, z\right)+s d\left(x^{*}, z\right)\right] \rightarrow 0$ as $n \rightarrow \infty$.

Hence, we get that $x^{*}=y^{*}$.

In a similar way, we can treat the rest of the cases. The proof is now complete.

It is worth to mention now that if we consider the coupled fixed point problem (1.1) in a complete $b$-metric space and we assume that the maximum-symmetric contraction condition on $T$ holds on $X \times X$, then the following theorem can be deduced. Notice that, in this case, we can relax the condition on the contraction constant to $k<1$.

Theorem 5. Let $(X, d)$ be a complete b-metric space with constant $s \geq 1$. Let $T: X \times X \rightarrow X$ be an operator. Assume that there exists $k \in(0,1)$ such that, for all $(x, y),(u, v) \in X \times X$, we have

$$
\max \{d(T(x, y), T(u, v)), d(T(y, x), T(v, u))\} \leq k \max \{d(x, u), d(y, v)\} .
$$

Then, the following conclusions hold:

(a) there exists a unique solution $\left(x^{*}, y^{*}\right) \in X \times X$ of the coupled fixed point problem (1.1), and, for any initial point $\left(x_{0}, y_{0}\right) \in X \times X$, the sequences $\left(x_{n}\right)_{n \in \mathbb{N}}$, $\left(y_{n}\right)_{n \in \mathbb{N}}$ defined, for $n \in \mathbb{N}$, by

$$
\left\{\begin{array}{c}
x_{n+1}=T\left(x_{n}, y_{n}\right) \\
y_{n+1}=T\left(y_{n}, x_{n}\right),
\end{array}\right.
$$

converge to $x^{*}$ and respectively to $y^{*}$ as $n \rightarrow \infty$.

(b) In particular, if $k<\frac{1}{s}$ and the $b$-metric $d$ is continuous, then, for all $n \in \mathbb{N}^{*}$, the following estimation holds

$$
\max \left\{d\left(T^{n}\left(x_{0}, y_{0}\right), x^{*}\right), d\left(T^{n}\left(y_{0}, x_{0}\right), y^{*}\right)\right\} \leq \frac{s k^{n}}{1-s k} \cdot \max \left\{d\left(x_{0}, T\left(x_{0}, y_{0}\right)\right), d\left(y_{0}, T\left(y_{0}, x_{0}\right)\right)\right\} .
$$

Proof. We introduce on $Z:=X \times X$ the functional $\tilde{d}: Z \times Z \rightarrow \mathbb{R}_{+}$defined by

$$
\tilde{d}((x, y),(u, v)):=\max \{d(x, u), d(y, v)\} .
$$

Notice that, as before, $\tilde{d}$ is a $b$-metric on $Z$ with the same constant $s \geq 1$ and, if the space $(X, d)$ is complete, then $(Z, \tilde{d})$ is complete too.

We consider now the operator $F: Z \rightarrow Z$ given by

$$
F(x, y):=(T(x, y), T(y, x)) .
$$

It is easy to prove now that $F$ is a contraction in $(Z, \tilde{d})$ with constant $k \in(0,1)$, i.e.,

$$
\tilde{d}(F(z), F(w)) \leq k \tilde{d}(z, w) \text {, for all } z, w \in Z \text {. }
$$

The first conclusion follows now by applying the $b$-metric space version of the contraction principle, see [5] or Theorem 12.2, page 115 in [9]. For the estimation, we can repeat the arguments in our previous existence theorems. 
Remark 3. We notice that, using Theorem 1 and the same method as before, we can study the following coupled coincidence problem:

$$
\left\{\begin{array}{l}
g(x)=T(x, y) \\
g(y)=T(y, x)
\end{array}\right.
$$

\section{A THEORY OF THE COUPLED FIXED POINT SET FOR MAX-SYMMETRIC}

\section{CONTRACTIONS}

In this section, we will study some qualitative properties of the coupled fixed point set related to a symmetric contraction condition, such as: data dependence, well-posedness, Ulam-Hyers stability and limit shadowing property.

For the data dependence problem, we have the following result.

Theorem 6. Let $(X, d)$ be a complete b-metric space with constant $s \geq 1$. Let $T_{i}: X \times X \rightarrow X(i \in\{1,2\})$ be two mappings. Assume that the following conditions are satisfied:

(i) there exists $k \in\left(0, \frac{1}{s}\right)$ such that, for all $(x, y),(u, v) \in X \times X$, we have

$\max \left\{d\left(T_{1}(x, y), T_{1}(u, v)\right), d\left(T_{1}(y, x), T_{1}(v, u)\right)\right\} \leq k \max \{d(x, u), d(y, v)\} ;$

(ii) the operator $T_{2}$ has at least one coupled fixed point in $X \times X$;

(iii) there exists $\eta>0$ such that

$$
d\left(T_{1}(x, y), T_{2}(x, y)\right) \leq \eta, \text { for all }(x, y) \in X \times X .
$$

In the above conditions, if $\left(x^{*}, y^{*}\right)$ denotes the unique coupled fixed point for $T_{1}$, then

$$
\max \left\{d\left(x^{*}, \bar{x}\right), d\left(y^{*}, \bar{y}\right)\right\} \leq \frac{s \eta}{1-s k}, \forall(\bar{x}, \bar{y}) \in C F i x\left(T_{2}\right) .
$$

Proof. Since the operator $T_{1}$ satisfies the hypotheses of Theorem 5, there exists a unique coupled fixed point for $T_{1}$, say $\left(x^{*}, y^{*}\right)$. Let $(\bar{x}, \bar{y}) \in C F i x\left(T_{2}\right)$. We consider again the $b$-metric $\tilde{d}: Z \times Z \rightarrow \mathbb{R}_{+}$defined by

$$
\tilde{d}((x, y),(u, v)):=\max \{d(x, u), d(y, v)\} .
$$

Then, we have:

$$
\begin{gathered}
\tilde{d}\left(\left(x^{*}, y^{*}\right),(\bar{x}, \bar{y})\right)=\tilde{d}\left(\left(T_{1}\left(x^{*}, y^{*}\right), T_{1}\left(y^{*}, x^{*}\right)\right),\left(T_{2}(\bar{x}, \bar{y}), T_{2}(\bar{y}, \bar{x})\right)\right) \leq \\
s\left(\tilde{d}\left(\left(T_{1}\left(x^{*}, y^{*}\right), T_{1}\left(y^{*}, x^{*}\right)\right),\left(T_{1}(\bar{x}, \bar{y}), T_{1}(\bar{y}, \bar{x})\right)\right)+\tilde{d}\left(T_{1}(\bar{x}, \bar{y}), T_{1}(\bar{y}, \bar{x})\right),\left(T_{2}(\bar{x}, \bar{y}), T_{2}(\bar{y}, \bar{x})\right)\right) \leq \\
s\left(k \max \left\{d\left(\left(x^{*}, \bar{x}\right),\left(y^{*}, \bar{y}\right)\right)\right\}+\eta\right)=s\left(k \tilde{d}\left(\left(x^{*}, y^{*}\right),(\bar{x}, \bar{y})\right)+\eta\right) .
\end{gathered}
$$

Thus,

and the proof is complete.

$$
\tilde{d}\left(\left(x^{*}, y^{*}\right),(\bar{x}, \bar{y})\right) \leq \frac{s \eta}{1-s k},
$$


We will study the well-posedness of the coupled fixed point problem (1.1).

Definition 3. Let $(X, d)$ be a $b$-metric space with constant $s \geq 1$ and $T: X \times X \rightarrow$ $X$ be an operator. By definition, the coupled fixed point problem (1.1) is said to be well-posed if:

(i) $\operatorname{CFix}(T)=\left\{\left(x^{*}, y^{*}\right)\right\}$;

(ii) for any sequence $\left(x_{n}, y_{n}\right)_{n \in \mathbb{N}}$ in $X \times X$ for which $d\left(x_{n}, T\left(x_{n}, y_{n}\right)\right) \rightarrow 0$ and respectively $d\left(y_{n}, T\left(y_{n}, x_{n}\right)\right) \rightarrow 0$ as $n \rightarrow \infty$, we have that $\left(x_{n}\right) \rightarrow x^{*}$ and $\left(y_{n}\right) \rightarrow$ $y^{*}$ as $n \rightarrow \infty$.

A well-posedness result is given in the following theorem.

Theorem 7. Assume that all the hypotheses of Theorem 5 take place. Additionally, assume that the contraction constant $k$ of $T$ satisfies the condition $k<\frac{1}{s}$. Then the coupled fixed point problem (1.1) is well-posed.

Proof. By Theorem 5 we get that $\operatorname{CFix}(T)=\left\{\left(x^{*}, y^{*}\right)\right\}$. Let $\left(x_{n}, y_{n}\right)_{n \in \mathbb{N}}$ be a sequence in $X \times X$ for which $d\left(x_{n}, T\left(x_{n}, y_{n}\right)\right) \rightarrow 0$ and respectively $d\left(y_{n}, T\left(y_{n}, x_{n}\right)\right)$ $\rightarrow 0$ as $n \rightarrow \infty$. As before, we will work on $Z:=X \times X$ with the metric $\tilde{d}$ given above. Then, we have:

$$
\begin{gathered}
\tilde{d}\left(\left(x_{n}, y_{n}\right),\left(x^{*}, y^{*}\right)\right)=\tilde{d}\left(\left(x_{n}, y_{n}\right),\left(T\left(x^{*}, y^{*}\right), T\left(y^{*}, x^{*}\right)\right)\right) \leq \\
s\left(\tilde{d}\left(\left(x_{n}, y_{n}\right),\left(T\left(x_{n}, y_{n}\right), T\left(y_{n}, x_{n}\right)\right)\right)+\tilde{d}\left(\left(T\left(x_{n}, y_{n}\right), T\left(y_{n}, x_{n}\right)\right),\left(T\left(x^{*}, y^{*}\right), T\left(y^{*}, x^{*}\right)\right)\right)\right) \leq \\
s\left(\max \left\{d\left(x_{n}, T\left(x_{n}, y_{n}\right)\right), d\left(y_{n}, T\left(y_{n}, x_{n}\right)\right)\right\}+k \max \left\{d\left(x_{n}, x^{*}\right), d\left(y_{n}, y^{*}\right)\right\}\right) .
\end{gathered}
$$

Thus

$$
\tilde{d}\left(\left(x_{n}, y_{n}\right),\left(x^{*}, y^{*}\right)\right) \leq \frac{s}{1-s k} \max \left\{d\left(x_{n}, T\left(x_{n}, y_{n}\right)\right), d\left(y_{n}, T\left(y_{n}, x_{n}\right)\right)\right\} \rightarrow 0 \text { as } n \rightarrow \infty \text {. }
$$

Hence, we get that $\left(x_{n}\right) \rightarrow x^{*}$ and $\left(y_{n}\right) \rightarrow y^{*}$ as $n \rightarrow \infty$.

We will consider the Ulam-Hyers stability of the coupled fixed point problem (1.1).

Definition 4. Let $(X, d)$ be a $b$-metric space with constant $s \geq 1$ and $T: X \times X \rightarrow$ $X$ be an operator. Let $\tilde{d}$ be any $b$-metric on $X \times X$ generated by $d$. By definition, the coupled fixed point problem (1.1) is said to be Ulam-Hyers stable if there exists an increasing operator $\psi: \mathbb{R}_{+} \rightarrow \mathbb{R}_{+}$, continuous in 0 with $\psi(0)=0$, such that for each $\varepsilon \in \mathbb{R}_{+}^{*}$ and for each solution $(\bar{x}, \bar{y}) \in X \times X$ of the inequality

$$
\tilde{d}((x, y),(T(x, y), T(y, x)) \leq \varepsilon
$$

there exists a solution $\left(x^{*}, y^{*}\right) \in X \times X$ of the coupled fixed point problem (1.1) such that

$$
\tilde{d}\left(\left(x^{*}, y^{*}\right),(\bar{x}, \bar{y})\right) \leq \psi(\varepsilon) .
$$

An Ulam-Hyers stability result for the coupled fixed point problem (1.1) is given in the following theorem. 
Theorem 8. Assume that all the hypotheses of Theorem 5 take place. Additionally, assume that the contraction constant $k$ of $T$ satisfies the condition $k<\frac{1}{s}$. Then the coupled fixed point problem (1.1) is Ulam-Hyers stable.

Proof. By Theorem 5 we get that $\operatorname{CFix}(T)=\left\{\left(x^{*}, y^{*}\right)\right\}$. Let any $\varepsilon>0$ and let $(\bar{x}, \bar{y}) \in X \times X$ such that

$$
\max \{d(\bar{x}, T(\bar{x}, \bar{y})), d(\bar{y}, T(\bar{y}, \bar{x}))\} \leq \varepsilon .
$$

Then, working on $Z:=X \times X$ with the $b$-metric $\tilde{d}$, we have

$$
\begin{gathered}
\tilde{d}\left((\bar{x}, \bar{y}),\left(x^{*}, y^{*}\right)\right)=\tilde{d}\left((\bar{x}, \bar{y}),\left(T\left(x^{*}, y^{*}\right), T\left(y^{*}, x^{*}\right)\right)\right) \leq \\
s\left(\tilde{d}((\bar{x}, \bar{y}),(T(\bar{x}, \bar{y}), T(\bar{y}, \bar{x})))+\tilde{d}\left((T(\bar{x}, \bar{y}), T(\bar{y}, \bar{x})),\left(T\left(x^{*}, y^{*}\right), T\left(y^{*}, x^{*}\right)\right)\right)\right) \leq \\
s\left(\max \{d(\bar{x}, T(\bar{x}, \bar{y})), d(\bar{y}, T(\bar{y}, \bar{x}))\}+k \max \left\{d\left(\bar{x}, x^{*}\right), d\left(\bar{y}, y^{*}\right)\right\}\right)
\end{gathered}
$$

Thus

which leads to our conclusion.

$$
\tilde{d}\left((\bar{x}, \bar{y}),\left(x^{*}, y^{*}\right)\right) \leq \frac{s}{1-s k} \varepsilon,
$$

The so-called limit shadowing property will be discussed now for the case of a coupled fixed point problem.

Definition 5. Let $(X, d)$ be a $b$-metric space with constant $s \geq 1$ and $T: X \times$ $X \rightarrow X$ be an operator. By definition, the coupled fixed point problem (1.1) has the limit shadowing property if, for any sequence $\left(x_{n}, y_{n}\right)_{n \in \mathbb{N}}$ in $X \times X$ for which $d\left(x_{n+1}, T\left(x_{n}, y_{n}\right)\right) \rightarrow 0$ and respectively $d\left(y_{n+1}, T\left(y_{n}, x_{n}\right)\right) \rightarrow 0$ as $n \rightarrow \infty$, there exists a sequence $\left(T^{n}(x, y), T^{n}(y, x)\right)_{n \in \mathbb{N}}$ such that $d\left(x_{n}, T^{n}(x, y)\right) \rightarrow 0$ and $d\left(y_{n}, T^{n}(y, x)\right) \rightarrow 0$ as $n \rightarrow \infty$.

A shadowing type result for the coupled fixed point problem is the following result.

Theorem 9. Assume that all the hypotheses of Theorem 5 take place. Additionally, assume that the contraction constant $k$ of $T$ satisfies the condition $k<\frac{1}{s}$. Then the coupled fixed point problem (1.1) for $T$ has the limit shadowing property.

Proof. By Theorem 5 we know that $\operatorname{CFix}(T)=\left\{\left(x^{*}, y^{*}\right)\right\}$ and, for any initial starting point $(x, y) \in X \times X$, we have that $\left(T^{n}(x, y)\right) \rightarrow x^{*}$ and $\left(T^{n}(y, x)\right) \rightarrow y^{*}$ as $n \rightarrow \infty$. Let $\left(x_{n}, y_{n}\right)_{n \in \mathbb{N}}$ be a sequence in $X \times X$ such that

$$
d\left(x_{n+1}, T\left(x_{n}, y_{n}\right)\right) \rightarrow 0 \text { and } d\left(y_{n+1}, T\left(y_{n}, x_{n}\right)\right) \rightarrow 0,
$$

as $n \rightarrow \infty$. We consider again on $Z:=X \times X$ the $b$-metric $\tilde{d}$. Then, for every $(x, y) \in X \times X$, we have

$$
\begin{gathered}
\tilde{d}\left(\left(x_{n+1}, y_{n+1}\right),\left(T^{n+1}(x, y), T^{n+1}(y, x)\right)\right) \leq \\
s\left[\tilde{d}\left(\left(x_{n+1}, y_{n+1}\right),\left(x^{*}, y^{*}\right)\right)+\tilde{d}\left(\left(x^{*}, y^{*}\right),\left(T^{n+1}(x, y), T^{n+1}(y, x)\right)\right)\right] .
\end{gathered}
$$


For the first term of the above sum, we can write

$$
\begin{gathered}
\tilde{d}\left(\left(x_{n+1}, y_{n+1}\right),\left(x^{*}, y^{*}\right)\right) \leq \\
s\left[\tilde{d}\left(\left(x_{n+1}, y_{n+1}\right),\left(T\left(x_{n}, y_{n}\right), T\left(y_{n}, x_{n}\right)\right)\right)+\tilde{d}\left(\left(T\left(x_{n}, y_{n}\right), T\left(y_{n}, x_{n}\right)\right),\left(T\left(x^{*}, y^{*}\right), T\left(y^{*}, x^{*}\right)\right)\right)\right] \leq \\
s \tilde{d}\left(\left(x_{n+1}, y_{n+1}\right),\left(T\left(x_{n}, y_{n}\right), T\left(y_{n}, x_{n}\right)\right)\right)+\operatorname{sk} \tilde{d}\left(\left(x_{n}, y_{n}\right),\left(x^{*}, y^{*}\right)\right) \leq \\
s \tilde{d}\left(\left(x_{n+1}, y_{n+1}\right),\left(T\left(x_{n}, y_{n}\right), T\left(y_{n}, x_{n}\right)\right)\right)+ \\
\operatorname{sk}\left[s \tilde{d}\left(\left(x_{n}, y_{n}\right),\left(T\left(x_{n-1}, y_{n-1}\right), T\left(y_{n-1}, x_{n-1}\right)\right)\right)+\operatorname{sk} \tilde{d}\left(\left(x_{n-1}, y_{n-1}\right),\left(x^{*}, y^{*}\right)\right)\right] \leq \\
\ldots \leq s \sum_{p=0}^{n}(s k)^{n-p} \tilde{d}\left(\left(x_{p+1}, y_{p+1}\right),\left(T\left(x_{p}, y_{p}\right), T\left(y_{p}, x_{p}\right)\right)\right)+(s k)^{n+1} \tilde{d}\left(\left(x_{0}, y_{0}\right),\left(x^{*}, y^{*}\right)\right) .
\end{gathered}
$$

The first therm of the above sum converges to zero by the Cauchy Lemma (see, for example [13]), while the second one goes to zero since $s k<1$.

Thus,

$$
\tilde{d}\left(\left(x_{n+1}, y_{n+1}\right),\left(T^{n+1}(x, y), T^{n+1}(y, x)\right)\right) \rightarrow 0 \text { as } n \rightarrow \infty,
$$

and the conclusion follows.

Remark 4. Notice that similar results (such as data dependence, well-posedness property, Ulam-Hyers stability, limit shadowing property) can be established in the framework of a metric space endowed with a partial order relation and under the max-symmetric contraction condition with respect to the comparable elements of the space and the mixed monotone assumption on $T$, but, in this case, some additional assumptions (involving comparison properties of some elements, see Theorem 2 in Section 3.) must be imposed.

\section{AN APPLICATION TO A PERIODIC BOUNDARY VALUE PROBLEM}

We will discuss now an application of the previous results to a periodic boundary value problem of the following type:

$$
\left\{\begin{array}{c}
x^{\prime}(t)=f(t, x(t))+g(t, x(t)), t \in(0, T) \\
x(0)=x(T)
\end{array}\right.
$$

A solution of the above problem is a function $x \in C^{1}[0, T]$ satisfying the above relations.

The above problem was considered for the first time in [7] and then in some related papers. We mention here the paper [3], where a nice improvement is given. We will follow the above mentioned paper for the terms of our problem.

Let $\lambda_{1}, \lambda_{2}>0$ such that:

$$
\left\{\begin{array}{c}
\ln \frac{2 e-1}{e} \leq\left(\lambda_{2}-\lambda_{1}\right) T \\
\left(\dot{\lambda}_{1}+\lambda_{2}\right) T \leq 1
\end{array}\right.
$$


In order to obtain the solution of (5.1) we study now the existence of a solution of the following periodic system of differential equations:

$$
\left\{\begin{array}{c}
x^{\prime}+\lambda_{1} x-\lambda_{2} y=f(t, x)+g(t, y)+\lambda_{1} x-\lambda_{2} y, t \in(0, T) \\
y^{\prime}+\lambda_{1} y-\lambda_{2} x=f(t, y)+g(t, x)+\lambda_{1} y-\lambda_{2} x \\
x(0)=x(T) \\
y(0)=y(T),
\end{array}\right.
$$

which is equivalent to the following system of integral equations for $t \in[0, T]$

$\left\{\begin{array}{l}x(t)=\int_{0}^{T} G_{1}(t, s)\left[f(s, x)+g(s, y)+\lambda_{1} x-\lambda_{2} y\right]+G_{2}(t, s)\left[f(s, y)+g(s, x)+\lambda_{1} y-\lambda_{2} x\right] d s \\ y(t)=\int_{0}^{T} G_{1}(t, s)\left[f(s, y)+g(s, x)+\lambda_{1} y-\lambda_{2} x\right]+G_{2}(t, s)\left[f(s, x)+g(s, y)+\lambda_{1} x-\lambda_{2} y\right] d s,\end{array}\right.$

where $G_{1}, G_{2}$ have the same expressions like in [7]. By the assumptions (5.2), we obtain (see [7]) that $G_{1}(t, s) \geq 0$ and $G_{2}(t, s) \leq 0$, for all $t, s \in[0, T]$.

Then, we have the following existence and uniqueness result for the periodic system (5.3).

Theorem 10. Consider the periodic system of differential equations (5.3). We suppose:

(i) $f:[0, T] \times \mathbb{R} \rightarrow \mathbb{R}$ and $g:[0, T] \times \mathbb{R} \rightarrow \mathbb{R}$ are continuous;

(ii) there exists $\lambda_{1}, \lambda_{2}, \mu_{1}, \mu_{2}>0$ such that, for every $x, y \in \mathbb{R}$ with $y \leq x$, we have

$$
\begin{gathered}
0 \leq\left(f(t, x)+\lambda_{1} x\right)-\left(f(t, y)+\lambda_{1} y\right) \leq \mu_{1}(x-y) \\
-\mu_{2}(x-y) \leq\left(g(t, x)-\lambda_{2} x\right)-\left(g(t, y)-\lambda_{2} y\right) \leq 0,
\end{gathered}
$$

where

$$
\left(\frac{\mu_{1}+\mu_{2}}{\lambda_{1}+\lambda_{2}}\right)^{2}+\left(\frac{\mu_{1}-\mu_{2}}{\lambda_{1}-\lambda_{2}}\right)^{2}<\frac{1}{2}
$$

(iii) the relations (5.2) are satisfied;

(iv) there exist lower and upper solution $x_{0}, y_{0} \in C[0, T]$ for the system (5.3) such that

$$
\left\{\begin{array}{l}
\lambda_{1}\left(x_{0}(T)-x_{0}(0)\right)+\lambda_{2}\left(y_{0}(0)-y_{0}(T)\right) \leq \frac{x_{0}(T)-x_{0}(0)}{T} \\
\lambda_{1}\left(y_{0}(T)-y_{0}(0)\right)+\lambda_{2}\left(x_{0}(0)-x_{0}(T)\right) \leq \frac{y_{0}(T)-y_{0}(0)}{T}
\end{array}\right.
$$

Then, there exists at least one solution $\left(x^{*}, y^{*}\right)$ of the system (5.3).

Proof. We will work in the $b$-metric space with constant $s=2(X, d)$, with $X:=$ $C[0, T]$ and

$$
d(x, y):=\max _{t \in[0, T]}\left((x(t)-y(t))^{2}\right)=\left\|(x-y)^{2}\right\|_{C},
$$

where $\|\cdot\|_{C}$ denotes the classical Chebyshev norm. 
We can prove that all the assumptions of Theorem 2 are satisfied. We define $S$ : $X \times X \rightarrow X$ by

$S(x, y)(t):=\int_{0}^{T}\left(G_{1}(t, s)\left[f(s, x)+g(s, y)+\lambda_{1} x-\lambda_{2} y\right]+G_{2}(t, s)\left[f(s, y)+g(s, x)+\lambda_{1} y-\lambda_{2} x\right]\right) d s$.

Then, the system (5.3) can be written as a coupled fixed point problem for $S$ :

$$
\left\{\begin{array}{l}
x=S(x, y) \\
y=S(y, x) .
\end{array}\right.
$$

By (ii), it follows, in a similar way to [7], that $S$ satisfies the mixed monotone condition.

By (iv), we have that $x_{0}(t) \leq S\left(x_{0}, y_{0}\right)(t)$ and $y_{0}(t) \geq S\left(y_{0}, x_{0}\right)(t)$, for all $t \in[0, T]$.

Moreover, for $x \leq u, y \geq v$, we can write

$$
\begin{gathered}
(S(x, y)(t)-S(u, v)(t))^{2}= \\
\left(\int_{0}^{T} G_{1}(t, s)\left[\left(f(s, x)+g(s, y)+\lambda_{1} x-\lambda_{2} y\right)-\left(f(s, u)+g(s, v)+\lambda_{1} u-\lambda_{2} v\right)\right]-\right. \\
\left.G_{2}(t, s)\left[\left(f(s, v)+g(s, u)+\lambda_{1} v-\lambda_{2} u\right)-\left(f(s, y)+g(s, x)+\lambda_{1} y-\lambda_{2} x\right)\right] d s\right)^{2} \leq \\
\left(\int_{0}^{T} G_{1}(t, s)\left[\mu_{1}(u-x)+\mu_{2}(y-v)\right]-G_{2}(t, s)\left[\mu_{1}(y-v)+\mu_{2}(u-x)\right] d s\right)^{2}= \\
\left(\int_{0}^{T}\left(\mu_{1} G_{1}(t, s)-\mu_{2} G_{2}(t, s)\right) \sqrt{(u-x)^{2}} d s+\int_{0}^{T}\left(\mu_{2} G_{1}(t, s)-\mu_{1} G_{2}(t, s)\right) \sqrt{(y-v)^{2}} d s\right)^{2} \leq \\
2\left(\left[\int_{0}^{T}\left(\mu_{1} G_{1}(t, s)-\mu_{2} G_{2}(t, s)\right) \sqrt{(u-x)^{2}} d s\right]^{2}+\left[\int_{0}^{T}\left(\mu_{2} G_{1}(t, s)-\mu_{1} G_{2}(t, s)\right) \sqrt{(y-v)^{2}} d s\right]^{2}\right) \leq \\
2\left[\left(\int_{0}^{T}\left(\mu_{1} G_{1}(t, s)-\mu_{2} G_{2}(t, s)\right) d s\right)^{2}+\left(\int_{0}^{T}\left(\mu_{2} G_{1}(t, s)-\mu_{1} G_{2}(t, s)\right) d s\right)^{2}\right] \\
\max \left\{\left\|(x-u)^{2}\right\|_{C},\left\|(y-v)^{2}\right\|_{C}\right\}= \\
{\left[\left(\frac{\mu_{1}+\mu_{2}}{\lambda_{1}+\lambda_{2}}\right)^{2}+\left(\frac{\mu_{1}-\mu_{2}}{\lambda_{1}-\lambda_{2}}\right)^{2}\right] \cdot \max \{d(x, u), d(y, v)\} . \forall t \in[0, T] .}
\end{gathered}
$$

Thus,

$d(S(x, y), S(u, v)) \leq k \max \{d(x, u), d(y, v)\}$, where $k:=\left(\frac{\mu_{1}+\mu_{2}}{\lambda_{1}+\lambda_{2}}\right)^{2}+\left(\frac{\mu_{1}-\mu_{2}}{\lambda_{1}-\lambda_{2}}\right)^{2}$.

By a similar approach, we get that $d(S(y, x), S(v, u)) \leq k \max \{d(x, u), d(y, v)\}$.

Hence, for all $x \leq u, y \geq v$, we get

$$
\max \{d(S(x, y), S(u, v)), d(S(y, x), S(v, u))\} \leq k \max \{d(x, u), d(y, v)\} .
$$

Since $k<\frac{1}{2}$, all the assumptions of Theorem 2 are satisfied and the result follows by Theorem 2 . 
We will discuss now existence and uniqueness for the solution of the periodic boundary value problem (5.1).

Theorem 11. Consider the periodic boundary value problem (5.1). Suppose that all the assumptions of Theorem 10 are satisfied. Then, the periodic boundary value problem (5.1) has a unique solution $x^{*}$.

Proof. Notice that the space $X:=C[0, T]$ endowed with the partial order relation

$$
x \leq_{C} y \Longleftrightarrow x(t) \leq y(t) \text { for all } t \in[0, T],
$$

has the property that every pair of elements of $X$ has a lower bound or an upper bound. Thus, we can apply Theorem 3 and Theorem 4 , and we get that $\left(x^{*}, y^{*}\right)$ is unique and $x^{*}=y^{*}$. Hence, $x^{*}$ is the unique solution of the periodic boundary value problem (5.1).

\section{REFERENCES}

[1] I. Bakhtin, "The contraction mapping principle in almost metric spaces," Funct. Anal., vol. 30, no. 1, pp. 26-37, 1989.

[2] V. Berinde, "Generalized contractions in quasimetric spaces," Seminar on Fixed Point Theory Cluj-Napoca, vol. 1993, pp. 3-9, 1993.

[3] V. Berinde, "Generalized coupled fixed point theorems for mixed monotone mappings in partially ordered metric spaces," Nonlinear Anal., vol. 74, no. 18, pp. 7347-7355, 2011, doi: 10.1016/j.na.2011.07.053.

[4] L. Blumenthal, Theory and Applications of Distance Geometry. Oxford: Oxford, Clarendon Press, 1953. doi: 10.1090/S0002-9904-1954-09800-2.

[5] S. Czerwik, "Contraction mappings in b-metric spaces," Acta Math. Inform. Univ. Ostraviensis, vol. 1, pp. 5-11, 1993.

[6] T. Gnana Bhaskar and V. Lakshmikantham, "Fixed point theorems in partially ordered metric spaces and applications," Nonlinear Anal., vol. 65, no. 7, pp. 1379-1393, 2006, doi: 10.1016/j.na.2005.10.017.

[7] D. Guo and V. Lakshmikantham, "Coupled fixed points of nonlinear operators with applications," Nonlinear Anal., vol. 11, no. 11, pp. 623-632, 1987, doi: 10.1016/0362-546X(87)90077-0.

[8] J. Heinonen, Lectures on Analysis on Metric Spaces. Berlin: Springer, 2001.

[9] W. Kirk and N. Shahzad, Fixed Point Theory in Distance Spaces. Heidelberg: Springer, 2014. doi: 10.1007/978-3-319-10927-5.

[10] V. Lakshmikantham and L. Ciric, "Coupled fixed point theorems for nonlinear contractions in partially ordered metric spaces," Nonlinear Anal., vol. 70, no. 12, pp. 4341-4349, 2009.

[11] V. Opoitsev and T. Khurodze, Nonlinear operators in spaces with a cone, ser. Tbilis. Gos. Univ. Tbilisi. Tbilisi: Tbilis. Gos. Univ., 1984.

[12] A. Petrusel, G. Petrusel, B. Samet, and J. Yao, "Coupled fixed point theorems for symmetric contractions in b-metric spaces with applications to a system of integral equations and a periodic boundary value problem," Fixed Point Theory, vol. 17, no. 2, pp. 457-476, 2016.

[13] A. Petrusel and I. Rus, "The theory of a metric fixed point theorem for multivalued operators," in Fixed Point Theory and its Applications, ser. 9th Intern. Conf. on Fixed Point Theory and its Appl., L. Lin, A. Petrusel, and H. Xu, Eds. Yokohama: Yokohama Publ., 2010, pp. 161-175.

[14] A. Ran and M. Reurings, "A fixed point theorem in partially ordered sets and some applications to matrix equations," Proc. Amer. Math. Soc., vol. 132, no. 5, pp. 1435-1443, 2004. 
[15] B. Samet, "Coupled fixed point theorems for a generalized Meir-Keeler contraction in partially ordered metric spaces," Nonlinear Anal., vol. 72, no. 12, pp. 4508-4517, 2010, doi: 10.1016/j.na.2010.02.026.

[16] C. Urs, "Coupled fixed point theorems and applications," Miskolc Math. Notes, vol. 14, no. 1, pp. 323-333, 2013.

Authors' addresses

Adrian Petruşel

Department of Mathematics, Babeş-Bolyai University Cluj-Napoca,, Kogălniceanu Street No.1, 400084, Cluj-Napoca, Romania

E-mail address: petrusel@math.ubbcluj.ro

\section{Gabriela Petruşel}

Department of Business, Babeş-Bolyai University Cluj-Napoca,, Kogălniceanu Street No.1, 400084, Cluj-Napoca, Romania

E-mail address: gabi.petruseletbs.ubbcluj.ro

\section{Bessem Samet}

King Saud University, College of Science, Department of Mathematics, P.O. Box 2455, Riyadh 11451, Saudi Arabia

E-mail address: bsamet@ksu.edu.sa 